\title{
MEDICATION DURING PREGNANCY IN BELGIUM: LINKING THREE ADMINISTRATIVE DATABASES: CHALLENGES AND OPPORTUNITIES
}

\section{$\cdots$ AUTEURS}

Lionel LARCIN, Fati KIRAKOYA-SAMADOULOUGOU

Centre de recherche Épidémiologie, Biostatistiques, Recherche Clinique, School of Public Health, Université libre de Bruxelles (ULB), Brussels, Belgium

\section{> lionel.larcin@ulb.be}

\section{..... INTRODUCTION}

In Belgium there are currently not linked databases available to study the utilization of medication during pregnancy at the population level. We propose to link the prescription data from health insurers database, certificates of live births and deaths under one-year data from Statbel and data related to hospital stays due to pregnancies and childbirths from the Federal Public Health Service.

\section{...... AIM}

The objectives of this study were to determine whether linkage between the three administrative databases is technically feasible for the period (2010-2016) and to describe the record linkage method.

\section{..3 METHODS}

After a review of the literature on pregnancy studies that used linked databases, we assessed from each of the three Belgian databases potential useful information. Consultations with each database holder allowed us to identify a linking procedure respecting the legal regulations. We paid particular attention to identify pregnancies episodes, congenital malformations, mother-infant relationships, levels of medication exposure and potential confounders (e.g. comorbidities).

\section{? RESULTS}

It is possible to link information from databases because each person has a unique identifier in each database. However, it remains a challenge to link stillbirth events recorded in death certificates as there are no identifier of the mothers concerned. Linking mothers with stillbirths using a probabilistic linkage could partially solved this issue. Congenital anomalies included were only those detected at birth, but congenital anomalies could be discovered later. To capture more information, we add ICD-10 diagnostic codes of congenital anomalies registered in hospitals for up to 3 months after birth.
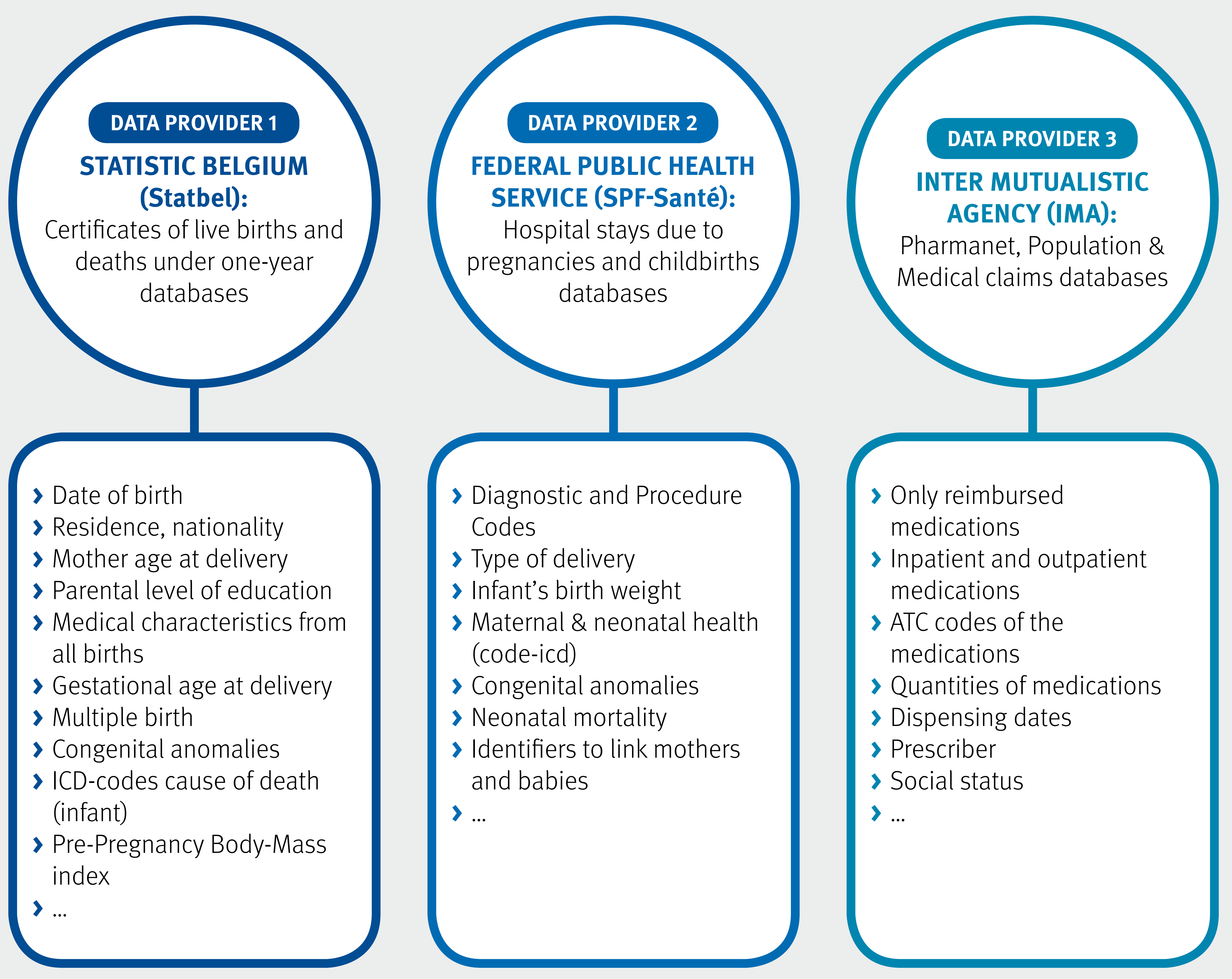

\section{IMPLEMENTATION:}

Trusted Third Party used pseudo-Ids to link data from different sources. Researchers access the linked \& pseudonymized data in a secure environment. $\ldots . . .3$ CONCLUSION
Linked databases combine information on pregnancies, socioeconomic status, medication use, the delivery, and infant health providing an excellent opportunity to study exposure-outcome associations for medication use during pregnancy. 